\title{
Formulation and evaluation of clindamycin HCL in situ gel for vaginal application
}

\author{
Priya Patel, Paresh Patel ${ }^{1}$ \\ Department of Pharmaceutical Sciences, Saurashtra University, Rajkot, 'Shivam Pharmaceutical Studies and Research Center, Valasan, \\ Gujarat, India
}

\begin{abstract}
Objective: The vagina has been studied as a favorable site for the local and systemic delivery of drugs, for female associated conditions. Vaginal preparations, although generally perceived as safer most still associated with number of problems including multiple days of dosing, dripping, leakage and messiness, causing discomfort to users and expulsion due to the self-cleansing action of the vaginal tract. These limitations lead to poor patient compliance and failure of the desired therapeutic effects. For efficient vaginal delivery of drugs, the delivery system should reside at the site of infection for a prolonged period of time. In situ gel formulation which combines advantages of both gels and solution so that an accurate dose can be administered with ease. These formulations remain in solution state before administration and transforms to gel after administration in to vaginal cavity. Material and Methods: In these formulations we prepared clindamycin loaded hydroxypropyl methycellulose $(0.1 \%)$ (bioadhesive) and gellan gum (ion activated gelling polymer) based in situ gel system for vaginal application. $\mathrm{NaCl}(0.9 \%)$ was added as an isotonic agent. The developed formulation was characterized for various in vitro parameters such as clarity, refractive index, $\mathrm{pH}$, viscosity, drug release profile, statistical release kinetics, bioadhesive force, and microbial efficacy along with stability studies. To simulate vaginal conditions, synthetic membrane (cellophane hydrated with modified simulated vaginal fluid) was used as model membranes. Results and Discussion: The developed formulation was found to be nonirritant, bioadhesive with good retention properties. Formulations have satisfactory appearance, clarity and drug content in the range 98.1-101\%. Refractive index of the gel is ranging from 1.335 to 1.337 , proofing the transparency of gel. Furthermore, formulation displayed $33.3 \%$ cumulative drug release after $2 \mathrm{~h} .67 .4 \%$ after $6 \mathrm{~h}$ and $98.9 \%$ after $12 \mathrm{~h}$. Conclusion: Developed formulation should be stable. Hence, formulation is thus a viable alternative to conventional vaginal dosage forms.
\end{abstract}

Key words: Clindamycin $\mathrm{HCl}$, gellan gum, hydroxypropyl methycellulose, in situ gel

\section{INTRODUCTION}

A hundred million individuals are infected with sexually transmitted diseases (STD) caused by human immunodeficiency virus, herpes simplex virus, human papilloma virus, and other pathogens. Women are facing the greatest risk of acquiring STD because of substantial mucosal exposure to seminal fluids, high prevalence of non-consensual and non-protective sex. Therefore,

\section{Address for correspondence:}

Prof. Priya Patel,

Department of Pharmaceutical Sciences, Saurashtra University,

Rajkot, Gujarat, India.

E-mail: patelpriyav@gmail.com

\begin{tabular}{|l|l|}
\hline \multicolumn{2}{|c|}{ Access this article online } \\
\hline Quick Response Code: & Website: \\
\hline & www.jpionline.org \\
\cline { 2 - 2 } & DOI: \\
\hline
\end{tabular}

it is highly recommendable to develop a female controlled drug delivery system containing microbicide as a barrier device against STD. ${ }^{[1]}$ Among the various routes of drug delivery, the vaginal route offers many advantages due to its large permeation area, rich vascularization, avoidance of first pass metabolism and relatively low enzymatic activity. It should also allow self-administration, with minimal interference with body functioning and daily life, and obtain high bioavailability with other medications. The avoidance of hepatic first-pass metabolism, a reduction in the incidence and severity of gastrointestinal side-effects, a decrease in hepatic side-effects of drugs such as steroids, and overcoming of pain, tissue damage, and probable infection observed with parental routes. ${ }^{[2]}$ The rate and extent of drug absorption after intravaginal administration may vary depending on formulation factors, vaginal physiology, age of the patient and menstrual cycle. Suppositories, creams, gels, tablets and vaginal rings are commonly used vaginal drug delivery systems. ${ }^{\left[{ }^{[3]}\right.}$

The conventional dosage forms such as preformed gel and solutions have limitations that they do not remain for long time at the site of application and needs frequent dosing. Direct 
application of gels onto the infected sites of the vagina might be difficult, inconvenient as well as have frequent dosing because the conventional gels do not remain for long time at the site of application. A new and recent approach is to try to combine advantages of both gels and solution so that an accurate dose can be administered with ease of administration. ${ }^{[4]}$ These formulations remain to a solution state before administration but transforms to gel after administration in to vaginal cavity. ${ }^{[5]}$

In situ gel has broad drug absorption peak and a longer drug residence time as compared to conventional dosage form. For a better therapeutic efficacy and patient compliance, mucoadhesive, thermosensitive and prolonged release vaginal gel was formulated for the treatment of vaginitis.

Nowadays, in situ-gelling liquids have also proved as more convenient dosage forms for local applications because they are easy to administer into desired body cavities. To achieve desirable therapeutic effect, vaginal delivery systems need to reside at the sites of infection for a prolonged period. The conventional formulations such as solutions, suspensions, ointments, etc., shows some constraints such as increased elimination, high variability in efficiency which reduces their bioavailability. In situ activated gel forming systems are liquid upon instillation and undergo phase transition in the vagina to form a viscoelastic gel in response to environmental changes such as change in temperature and $\mathrm{pH}$. Hence, it offers higher efficacy and bioavailability as compare to other conventional dosage form.

Some researcher explored efficacy of in situ vaginal gel. The liquid applied to topical areas turns into gels as a result of physical and/or chemical change induced by physiological environments such as $\mathrm{pH}$ forcellulose acetate phthalate, the concentration of calcium ions for gellan gum, temperature for poloxamers, etc. ${ }^{[6,7]}$ Bioadhesion and retention at the site of application for a sufficient period of time can be achieved by incorporating bioadhesive polymers in the formulations. Until date, only a limited number of studies have been reported on bioadhesive drug delivery systems for vaginal administration. Hydroxypropyl methycellulose (HPMC) act as bioadhesive. Gellan gum is an ion activated polymer, which gel when comes in contact with ions that is, vaginal fluid.

Clindamycin HCL is a semi synthetic antibiotic. Clindamycin inhibits bacterial protein synthesis at the level of the bacterial ribosome. The antibiotic binds preferentially to the $50 \mathrm{~S}$ ribosomal subunit and affects the process of peptide chain initiation. Clindamycin is indicated for the treatment of bacterial vaginosis (formerly referred to as Hemophilus vaginitis, Gardnerella vaginitis, nonspecific vaginitis, corynebacterium vaginitis, or anaerobic vaginosis) in nonpregnant women. ${ }^{[8]}$ Hence, in our present work, developing and optimizing a bioadhesive HPMC and gellan gum based in situ gel system of clindamycin for vaginal application. The optimized gel was evaluated for various physicochemical properties, in vitro drug release, bioadhesive force, retention time, and microbial efficacy and stability studies.

\section{MATERIALS AND METHODS}

Clindamycin phosphate was obtained as gift samples from Glenmark Pharmaceuticals, Mumbai, India. HPMC Was obtained as a gift sample from colorcon, Goa, Gellan gum (Gelrite ${ }^{\circledR}$ CP Kelco, US) was obtained as a gift from Applied Biosciences, Mumbai, India. All other chemicals and solvents used were purchased from local suppliers and of analytical grade unless mentioned.

\section{Preparation of simulated vaginal fluid}

The simulated vaginal fluid (SVF) were prepared by mixing $3.51 \mathrm{gL}-1 \mathrm{NaCl}, 1.40 \mathrm{gL}-1 \mathrm{KOH}, 0.222 \mathrm{gL}-1 \mathrm{Ca}(\mathrm{OH}) 2,0.018$ gL-1 bovine serum albumin, $2 \mathrm{gL}-1$ lactic acid, $1 \mathrm{gL}-1$ acetic acid, $0.16 \mathrm{gL}-1$ glycerol, $0.4 \mathrm{gL}-1$ urea and $5 \mathrm{gL}-1$ glucose. $\mathrm{PH}$ of the mixture was adjusted to $4.5 \pm 0.02$ by using $0.1 \mathrm{M}$ $\mathrm{HCl}^{\left[{ }^{9]}\right.}$

\section{Preparation of in situ gel formulation}

The "Cold Method" was used for preparation with slight modifications. ${ }^{[8]}$ The weighed quantity of drug was dissolved in saline phosphate buffer in aseptic condition. Benzalkonium chloride added as a preservative at the same time. Individually the polymeric solution of HPMC and gellan gum was prepared and kept undisturbed for $24 \mathrm{~h}$ for proper mixing [Table 1]. Further the drug and polymeric solution was mixed properly and intended quantity of the isotonic agent was also added to it. Solution was transferred into amber colored bottle and sealed till further use and resulting solutions were sterilized by autoclave at $121^{\circ} \mathrm{C}$ for $20 \mathrm{~min}$ at $15 \mathrm{psi}$.

\section{EVALUATION PARAMETERS}

\section{Interaction studies \\ Drug excipients compatibility study}

Drug excipients compatibility study was performed for checking the compatibility between drug and polymers. Drug excipient compatibility studies were also carried out by Using Fourier transform infrared (FT-IR) spectroscopy (Thermo scientific, Japan) also done by using double beam ultraviolet (UV)-visible spectrophotometer (Shimadzu pharmaspec. UV-1800, Japan). Liquid solutions of HPMC, gellan gum and clindamycin HCL was prepared individually and in combinations and were autoclaved at $121^{\circ} \mathrm{C}$ for $20 \mathrm{~min}$ at $15 \mathrm{psi}$. The UV spectra were taken before and after autoclaving using double beam UV-visible spectrophotometer. Both spectra were compared for any possible

\section{Table 1: Composition of formulations}

\begin{tabular}{lcccccc}
\hline Ingredients & F1 \% & F2 \% & F3 \% & F4 \% & F5 \% & F6 \% \\
\hline Clindamycin HCL & 2 & 2 & 2 & 2 & 2 & 2 \\
HPMC (\% w/v) & 0.5 & 0.5 & 1 & 1 & - & 1.5 \\
Gellan gum (\% w/v) & 0.5 & 1 & 0.5 & 1 & 1.5 & - \\
Benzalkonium chloride & 0.02 & 0.02 & 0.02 & 0.02 & 0.02 & 0.02 \\
Sodium chloride & 0.9 & 0.9 & 0.9 & 0.9 & 0.9 & 0.9 \\
Purified water & Q.S & Q.S & Q.S & Q.S & Q.S & Q.S \\
\hline
\end{tabular}

HCL: Hydrochloride, HPMC: Hydroxypropyl methylcellulose 
change due to interactions between different ingredients. Also taken FT-IR spectra of drug, polymers and formulations.

\section{Physicochemical characterization}

Vagina is capable of self-cleaning and regularly secretes vaginal fluid with slowly flush to wash out unwanted waste and foreign material. One of the most challenging tests for vaginal drug delivery is bioadhesive property which helps to prolong the residence of formulation.

\section{pH evaluation}

The $\mathrm{pH}$ of the formulation was recorded with a $\mathrm{pH}$ meter (Mettler Instruments, Germany) and allowing equilibrating for $1 \mathrm{~min}$. Experiments were performed in triplicate.

\section{Viscosity measurements}

Viscosity of all formulated batches of in situ gel was measured by using Brookfield Viscometer (Brookfield Engineering Laboratories Inc., MA, USA) Using spindle no. T 97. Viscosity of in situ gelling solutions was measured at different angular velocities at temp. $37^{\circ} \mathrm{C}$. The tests were performed in triplicate.

\section{Gelation temperature}

Gelation temperature (GT) was measured by heating the formulation in a $15-\mathrm{mL}$ borosilicate glass test tube. Into each test tube, $2 \mathrm{~mL}$ of formulation solution were placed and heated with gentle stirring until the formulation solution gets gelled. Gel formation was considered as the point where there will be no flow when the test tubes were tilted $>90^{\circ} \mathrm{C}$.

\section{Clarity and refractive index}

The clarity of the formulations after and before gelling was determined by visual examination of the formulations under light alternatively against white and black backgrounds.

Refractive index of the formulations were determined by Abbe's refractometer. Switch on the monochromatic Na Light lamp and wait for 5 min to allow lamp warm up. Put the 1-2 drop of liquid between illuminating and measuring prism. Close the lower prism case. Use rotating knob to align the X-Mark in the eye piece with the shadow boundary separating the dark and bright area seen in the field of view. Read the refractive index from the scale.

\section{Texture evaluation and consistency}

Texture of the gel in terms of stickiness and grittiness was evaluated by using texture analysis. Texture analyzer equipped with a $5 \mathrm{~kg}$ load cell was used for mucoadhesion studies. ${ }^{[9]}$ Freshly excised bovine vaginal mucosa was frozen at $-30^{\circ} \mathrm{C}$. A section of $2 \mathrm{~mm}$ thickness was taken from the inner part of the surface of the frozen vaginal mucosa and attached to the lower end of the probe (P0.5 Perspex, $\theta: 12.5 \mathrm{~mm}$ ) of the instrument with cyanoacrylate glue. The mucosa was dipped into the vaginal mucus (frozen at $-30^{\circ} \mathrm{C}$ just after the excision and adjusted to $37^{\circ} \mathrm{C}$ during the experiment) and kept for $10 \mathrm{~min}$ prior to the commencement of the experiment. The gels were packed into $30 \mathrm{~mm}$ diameter tubes and centrifuged at 20,000 rpm for $10 \mathrm{~min}$ to remove the air bubbles from the gels and to ensure a smooth contact between the gels and the vaginal mucosa. The mucoadhesion studies were performed at $37^{\circ} \mathrm{C}$. The probe holding the vaginal mucosa was lowered onto the surface of the gel with a constant speed of $0.1 \mathrm{~mm} \mathrm{~s}-1$ and contact force of $0.5 \mathrm{~N}$ applied. After a contact period of $120 \mathrm{~s}$, the probe was then moved vertically upwards at a constant speed of $0.1 \mathrm{~mm} \mathrm{~s}-1$. Each experiment was carried out in triplicate.

\section{Gel persistent capacity and spreadability}

Gel persistent capacity was determined by placing drop of prepared formulation in vial containing $2 \mathrm{ml}$ of SVF and observed till it completely erodes. Spreadability was determined by wooden block and glass slide apparatus. Weights about $20 \mathrm{~g}$ were added to the pan and the time were noted for upper slide (movable) to separate completely from the fixed slides.

\section{Drug release}

The in vitro drug release study was performed in sink condition, using Franz diffusion cell (PermeGear, Inc. Bethlehem, PA) with water jacketed receptor chamber $(20 \mathrm{ml})$ and donor chamber thermostated at $37^{\circ} \mathrm{C}$. The receptor chamber was separated by cellulose membrane (Filter paper Whatman 41, 20-25 $\mu \mathrm{m}$, Whatman GmbH, Dassel, Germany) and each formulation was spread on the circular portion of the membrane. The drug release was measured by UV analysis method. The results of in vitro data were analyzed by statistical software to obtain the best fit kinetic model for in vitro drug release from optimized formulation. The test was conducted in triplicate.

\section{Bioadhesion measurement}

The assemblies developed for in vitro measurement of bioadhesive strength in a simulated vaginal environment are a modification of the previously reported bioadhesion test assembly. ${ }^{[9]}$ The method is based on the measurement of tensile strength or shear stress required to break the adhesive bond between a model membrane and the test formulation. The test formulation is sandwiched between two model membranes fixed on flexible supports in the assemblies for a sufficient period of time. After the adhesive bond has formed, the force (weight) required to separate the bond will be measured and calculated a bioadhesive strength.

\section{Microbiological studies}

The microbiological studies were carried out on the optimized formulation and $2 \% \mathrm{w} / \mathrm{v}$ of plain drug solution for comparison against micro-organism. Staphylococcus aureus will be used as the test microorganism. A layer of nutrient agar $(20 \mathrm{~mL})$ seeded with the test micro-organism $(0.2 \mathrm{~mL})$ was allowed to solidify in the petriplate. Cups were made on the solidified agar layer with the help of sterile borer at $4 \mathrm{~mm}$ diameter. Then volume of the formulations (optimized formulation and plain drug solution) containing equivalent amount of drug will be poured into the cups. After keeping petriplates at room temperature for $4 \mathrm{~h}$, the plates were incubated at $37^{\circ} \mathrm{C}$ for $24 \mathrm{~h}$. The zone of inhibition was observed. The diameter of zone of inhibition will be measured by an antibiotic zone finder. ${ }^{[10,11]}$ 


\section{Irritation test (Hen's Egg Test-chorioallantoic membrane test)}

For the present study, modified Hen's Egg Test-chorioallantoic membrane (HET-CAM) test was done as per method reported by Velpandian et al., 2006. ${ }^{[8]}$ The HET-CAM has been shown to be a method of assessing the potential irritancy of particular chemicals. The potential irritancy of compounds detected by observing adverse changes that occur in the chorionallantoic membrane of the egg after exposure to test chemicals. ${ }^{[9]}$ Briefly, fertilized hen's eggs were obtained from poultry farm. Three eggs for each formulation weighing between 50 and $55 \mathrm{~g}$ were selected. These eggs were incubated in humidified incubator at a temperature of $37^{\circ} \mathrm{C} \pm 0.5^{\circ} \mathrm{C}$ for 3 days.

On the day 3, egg albumin $(3 \mathrm{ml})$ was removed by using sterile techniques from the pointed end of the egg. The hole was sealed by $70 \%$ alcohol sterilized parafilm (American Can Company, USA) with the help of heated spatula. The eggs were kept in the equatorial position for the development of CAM away from the shell. The eggs were candled on the $5^{\text {th }}$ day of incubation and every day, thereafter non-viable embryos were removed. On the $10^{\text {th }}$ day formulations were instilled directly onto the CAM surface and left in contact for $5 \mathrm{~min}$. The membrane is examined for vascular damage and the time taken for injury to occur is recorded.

A $0.9 \% \mathrm{NaCl}$ solution was used as a control as it is reported to be practically non-irritant. The scores were recorded according to the scoring schemes as shown in below Table 2 .

\section{Stability studies}

Stability studies was carried out on optimized formulation according to ICH Guidelines for 3 months and after that checks all the physicochemical parameters of formulated clindamycin loaded in situ Gel.

\section{Table 2: Scoring chart for HET-CAM test}

\begin{tabular}{|c|c|c|}
\hline Effect & Score & Inference \\
\hline No visible hemorrhage & 0 & Nonirritant \\
\hline Just visible membrane discoloration & 1 & Mild irritant \\
\hline $\begin{array}{l}\text { Structures are covered partially due to } \\
\text { membrane discoloration or hemorrhage }\end{array}$ & 2 & $\begin{array}{l}\text { Moderately } \\
\text { irritant }\end{array}$ \\
\hline $\begin{array}{l}\text { Structures are covered totally due to } \\
\text { membrane discoloration or hemorrhages }\end{array}$ & 3 & $\begin{array}{l}\text { Severe } \\
\text { irritant }\end{array}$ \\
\hline
\end{tabular}

HET-CAM: Hen's Egg Test - Chorioallantoic Membrane

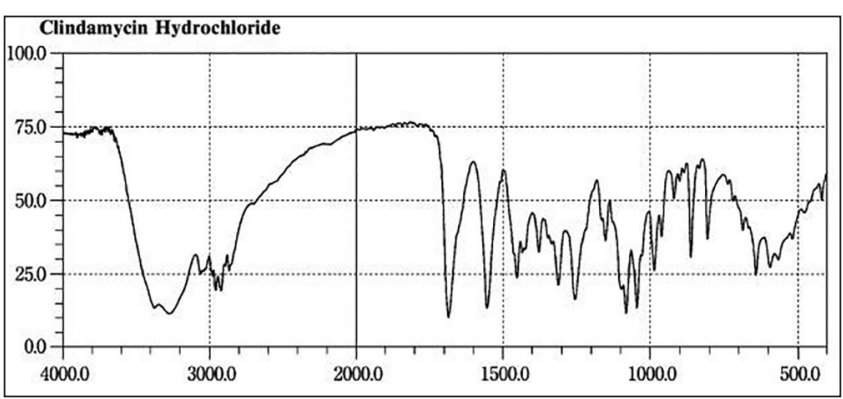

Figure 1: Fourier transform infrared spectra of clindamycin HCL

\section{RESULTS AND DISCUSSION}

Clindamycin HCL was scanned for its absorbance at $213 \mathrm{~nm}$ for generation of standard graph. Interaction studies were carried out to check any interaction between formulation ingredients. UV spectra obtained before and after autoclaving were found to be identical. No additional peak or shift in peak reveals two facts. First, the ingredients were compatible to each other and no physicochemical reactions took place, and secondly it also shows that the formulation can be terminally sterilized by autoclaving.

The FT-IR spectra of clindamycin HCL, HPMC, gellan gum and clindamycin loaded in situ formulation are depicted in Figures 1-4 respectively. FT-IR spectrum of clindamycin HCL showed characteristic peaks at $1755 \mathrm{~cm}^{-1}$ (C=O stretching), 1095 $\mathrm{cm}^{-1}$ (C-O stetching), $1516 \mathrm{~cm}^{-1}$ (C=C stretching), $2941 \mathrm{~cm}^{-1}$ (C-H stretching) whereas, FT-IR spectrum of HPMC showed characteristic peaks at $1729 \mathrm{~cm}^{-1} \mathrm{C}=\mathrm{O}$ (ester) stretching, 1448 and gellan gum shows $1482 \mathrm{CH}_{2}$ bending were identified, which was same in clindamycin loaded in situ formulation. Thus, there was no any interaction between drug and excipients.

Physicochemical evaluation of the prepared formulation The physicochemical properties such as $\mathrm{pH}$, gelation temperature, Viscosity, Reflective Index, Drug content, GPC (gel persistent spreadability [GPS]), and mucoadhesive strength of the formulations are depicted in Table 3.

$\mathrm{pH}$ of all the formulations were found to be in the range 5.3-5.5 that is, as per the $\mathrm{pH}$ of vagina. All developed Formulations have satisfactory appearance, clarity and drug content in the range 98.1-101\%.

$\mathrm{T}$ gel is the temperature at which the liquid phase makes a transition to gel. An ideal in situ gel should be a free flowing liquid at room temperature so as to allow reproducible administration into the site of application where it undergoes in situ phase transition to form a strong gel. ${ }^{[12]}$ The human vaginal temperature is $37.2^{\circ} \mathrm{C}$, ${ }^{[13]}$ So $\mathrm{T}$ gel of vaginal thermoreversible gels were considered to be suitable if they were in the range of $25-37^{\circ} \mathrm{C} .^{[12]}$ If the $\mathrm{T}$ gel is lower than $25^{\circ} \mathrm{C}$, a gel might be formed at room temperature leading to difficulties in manufacturing, handling, and administering. If $\mathrm{T}$ gel is higher than $37^{\circ} \mathrm{C}$, a liquid dosage form still exists at vaginal temperature, resulting in drainage of the formula from the vagina at an early stage.

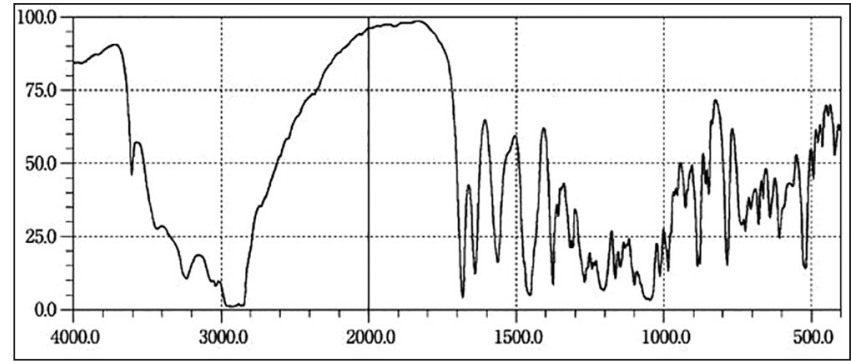

Figure 2: Fourier transform infrared spectra of optimized formulations (f-4) 


\begin{tabular}{|c|c|c|c|c|c|c|c|}
\hline $\begin{array}{l}\text { Formulation } \\
\text { code }\end{array}$ & $\mathrm{pH}$ & $\mathrm{GT}\left({ }^{\circ} \mathrm{C}\right)$ & $\begin{array}{l}\text { Viscosity } \\
\text { (cps) }\end{array}$ & $\begin{array}{l}\text { Drug } \\
\text { content (\%) }\end{array}$ & $\begin{array}{l}\text { Mucoadhesive } \\
\text { strength (dynes/cm) }\end{array}$ & $\begin{array}{l}\text { GPS } \\
\text { (h) }\end{array}$ & $\begin{array}{l}\text { Spreadability } \\
(\mathrm{mm})\end{array}$ \\
\hline F1 & 5.3 & 25.4 & $40 \pm 0.12$ & $94.3 \pm 0.06$ & + & 8 & 23 \\
\hline $\mathrm{F} 2$ & 5.3 & 33.8 & $48 \pm 0.42$ & $91.3 \pm 1.21$ & + & 9 & 25 \\
\hline F3 & 5.4 & 36.4 & $52 \pm 0.56$ & $89.2 \pm 0.34$ & ++ & 8 & 21 \\
\hline F4 & 5.3 & 36.8 & $56 \pm 0.12$ & $99.8 \pm 0.62$ & +++ & $>9$ & 14 \\
\hline F5 & 5.3 & 30.8 & $89 \pm 0.73$ & $99.3 \pm 0.24$ & + & 8 & 19 \\
\hline F6 & 5.4 & 35.9 & $90 \pm 0.18$ & $90.2 \pm 0.34$ & + & 8 & 17 \\
\hline
\end{tabular}

+: Poor/absent mucoadhesion strength, ++: Good mucoadhesion strength, +++: Excellent mucoadhesion strength, GPS: Gel persistent spreadability, GT: Gelation temperature

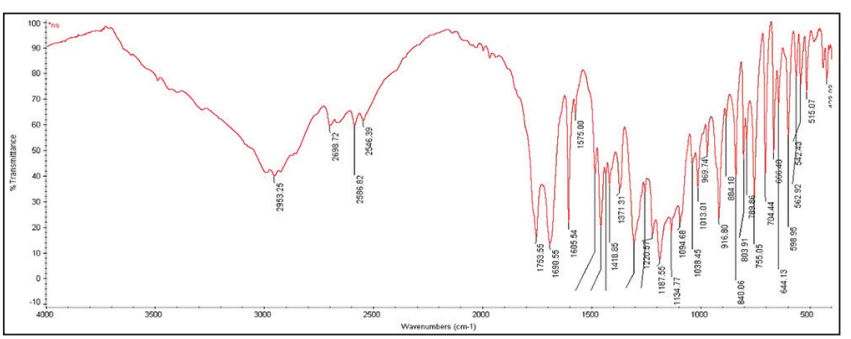

Figure 3: Fourier transform infrared spectra of hydroxypropyl methycellulose

Gel persistent spreadability were found having minimum $8 \mathrm{~h}$ and maximum $>9 \mathrm{~h}$ and optimized formulations was found $>9 \mathrm{~h}$.

Rheological analysis is a powerful technique to comprehensively investigate the gelation process and viscoelastic properties of thermo sensitive gel. The prepared formulations showed Newtonian flow as their viscosity is in $40-90$ cps range at formulation conditions. However the formulations showed increased viscosity in the SVF this behaviours desirable as it can promote good strength at various temperature and easy application. From the gelation temperature it was clear that the prepared formulation showed gelation at physiological condition. Addition of mucoadhesive polymer decrease the gelation temperature but it is near to body temperature. Gellan gum converted into stiff gel in the presence of ions and results in sudden increase in the viscosity.

The spreadability plays an important role in patient compliance and helps in uniform application of gel to the skin. A good gel takes less time to spread and will have high spreadability. Spreadability was found in range $14-25 \mathrm{~mm}$ optimized batch found $\mathrm{m} 14 \mathrm{~mm} \cdot{ }^{[14]}$

Refractive index of the gel is ranging from 1.335 to 1.337 , proofing the transparency of gel.

Bio adhesion and long retention are important and crucial physicochemical parameter for in situ forming vaginal gels of vaginal formulation. ${ }^{[15]} \mathrm{HPMC}$ act as a mucoadhesive, performed adhesion force studies proves the mucoadhesive nature of the HPMC. Gellan gum act as an ion activated polymer. Batch 5 and 6 having alone HPMC and gellan gum respectively. Hence these formulations do not give good mucoadhesive property. While in other hand Batch 4 having good mucoadhesive property due to the presence of both HPMC and gellan gum. Hence we can

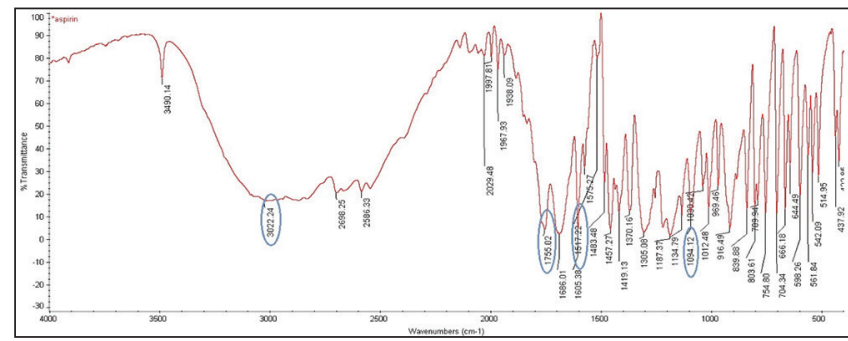

Figure 4: Fourier transform infrared spectra of gellan gum

conclude that both HPMC and gellan gum having a major role for in situ gel formulation for vaginal drug delivery. Accordingly synergistic effect of bioadhesive is predictable if gellan gum can be used with the widely use bioadhesive material like HPMC.

Clarity and texture analysis data was depicted in below Table 4. In case of all batches shown clear solutions and by texture analysis optimized batch shows nonsticky in nature. Hence, our optimized formulations suitable for vaginal drug delivery system.

\section{In vitro release kinetics}

In vitro drug release kinetics was carried out by the use of Franz diffusion cells in order to evaluate clindamycin in situ gel release profile. Initially the formulation demonstrates rapid release (burst effect) followed by slow and constant release for the rest of time was depicted in Figure 5. This pattern confirms the controlled release behavior of the formulation. The initial burst effect is beneficial as it help achieving the therapeutic concentration of drug in minimal time followed by constant release to maintain sustained and control release of the drug. Developed formulation displayed $33.3 \%$ cumulative drug release after 2 h. $67.4 \%$ after $6 \mathrm{~h}$ and $98.9 \%$ after $12 \mathrm{~h}$. Burst effect might be due to initial migration of the drug toward the surface of the matrix.

\section{Microbiological studies}

The optimized in situ gelling formulation showed antimicrobial activity when tested microbiologically by cup plate technique. Clear zone of inhibition were obtained. The diameter of zone of inhibition of optimized batch F4 having 18MM [Figure 6]. Its indicate optimized formulations inhibition of microbial growth.

\section{Irritation study}

Irritation of the developed formulation was checked by Hen's egg CAM test which is a rapid, sensitive and inexpensive test. ${ }^{[16]}$ Testing with incubated eggs is a borderline case between in vivo 
and in vitro systems and they does not conflict with the ethical and legal obligations.

Developed formulation was tested by using this method and result was compared with normal saline, which was used as control that is supoosed to be non-irritant because 0 score was obtained for normal saline. Chitosan/gellan gum based formulation was non-irritant up to $1 \mathrm{~h}$ (mean score 0 ) while the mean score was found to be 0.33 up to $24 \mathrm{~h}$ [Table 5]. The study shows that the formulation is non-irritant to mild irritant.

\section{Stability studies}

Stability study was carried out on the optimized formulation as per ICH guidelines for 5 months. There are no any major changes are observed in physicochemical characteristics as well as on release profile [Table 6 and Figure 7]. Hence, its indicates during stability test optimized formulations remain stable as per ICH Guideline. ${ }^{[16]}$

So, we can conclude that after the 3 months stability as per ICH Guidelines there are no any physicochemical changes are observed. Hence our formulation is stable.

\begin{tabular}{lll}
$\begin{array}{l}\text { Table 4: Clarity and texture analysis of prepared } \\
\text { formulations }\end{array}$ & \\
\hline Batch number & Clarity & Texture analysis \\
\hline F1 & Clear solution & Stiky \\
F2 & Clear solution & Stiky \\
F3 & Clear solution & Nonstiky \\
F4 & Clear solution & Nonstiky \\
F5 & Clear solution & Nonstiky \\
F6 & Clear solution & Nonstiky \\
\hline
\end{tabular}

\section{Table 5: Scores obtained in HET-CAM test}

\begin{tabular}{|c|c|c|c|c|c|c|c|c|c|}
\hline \multirow[t]{3}{*}{ Sample } & \multicolumn{9}{|c|}{ Score formulations } \\
\hline & \multicolumn{9}{|c|}{ Time (in min) } \\
\hline & 0 & 5 & 15 & 30 & 60 & 120 & 240 & 480 & 1440 \\
\hline \multicolumn{10}{|c|}{ Normal saline as control } \\
\hline Egg 1 & 0 & 0 & 0 & 0 & 0 & 0 & 0 & 0 & 0 \\
\hline Egg 2 & 0 & 0 & 0 & 0 & 0 & 0 & 0 & 0 & 0 \\
\hline Egg 3 & 0 & 0 & 0 & 0 & 0 & 0 & 0 & 0 & 0 \\
\hline Mean & 0 & 0 & 0 & 0 & 0 & 0 & 0 & 0 & 0 \\
\hline \multicolumn{10}{|c|}{ Developed formulations } \\
\hline Egg 1 & 0 & 0 & 0 & 0 & 0 & 0 & 0 & 0 & 0 \\
\hline Egg 2 & 0 & 0 & 0 & 0 & 0 & 0 & 0 & 0 & 1 \\
\hline Egg 3 & 0 & 0 & 0 & 0 & 0 & 1 & 1 & 1 & 1 \\
\hline Mean & 0 & 0 & 0 & 0 & 0 & 0.33 & 0.33 & 0.33 & 0.66 \\
\hline
\end{tabular}

Table 6: Physical evaluation of formulation after stability studies

\begin{tabular}{lccccc}
\hline $\begin{array}{l}\text { Formulation } \\
\text { code }\end{array}$ & pH & $\begin{array}{c}\text { GT } \\
\left({ }^{\circ} \mathbf{c}\right)\end{array}$ & $\begin{array}{c}\text { Viscosity } \\
\text { (cps) }\end{array}$ & $\begin{array}{c}\text { Drug } \\
\text { content } \\
\text { (\%) }\end{array}$ & $\begin{array}{c}\text { Mucoadhesive } \\
\text { strength } \\
\text { (dynes/cm) }\end{array}$ \\
\hline F4 (before) & 5.3 & 36.8 & $56 \pm 0.12$ & $99.8 \pm 0.62$ & +++ \\
F4 (after) & 5.3 & 36.7 & $55 \pm 0.32$ & $99.2 \pm 0.25$ & +++ \\
\hline
\end{tabular}

+++ Excellent mucoadhesion strength, GT: Gelation temperature

\section{CONCLUSION}

The vaginal route has been traditionally used for the conventional delivery of several locally acting drugs like antimicrobial agents. This study has described the in situ gel formulations of clindamycin HCL and evaluated their textural and rheological properties. Adding HPMC to the formulations decreased the sol to gel transition temperature, and affected the mucoadhesive, mechanical and rheological properties of the formulation. The results showed that the texture characterization was in agreement with rheological results confirming improved

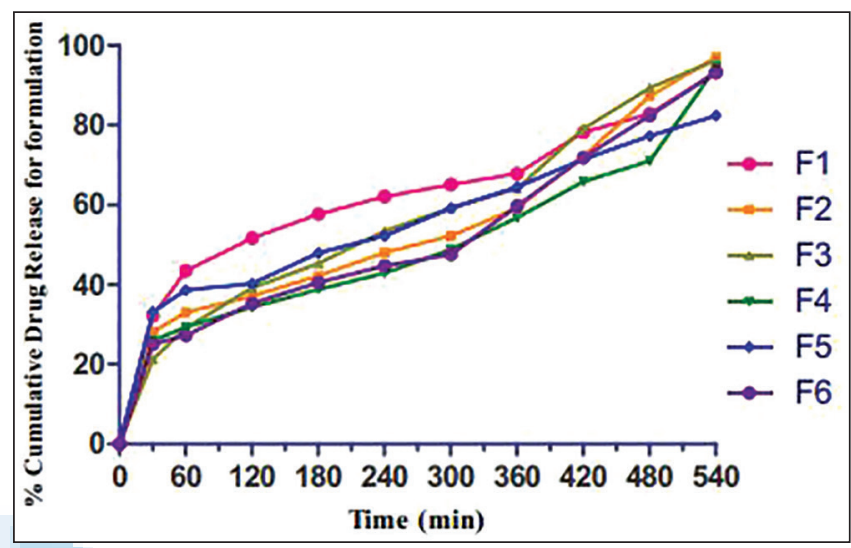

Figure 5: Drug release behaviour of prepared formulation

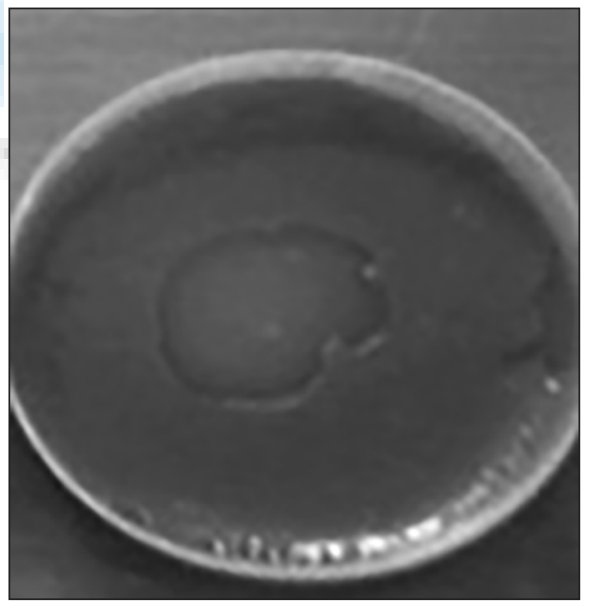

Figure 6: Zone of inhibition of optimized batch (f-4)

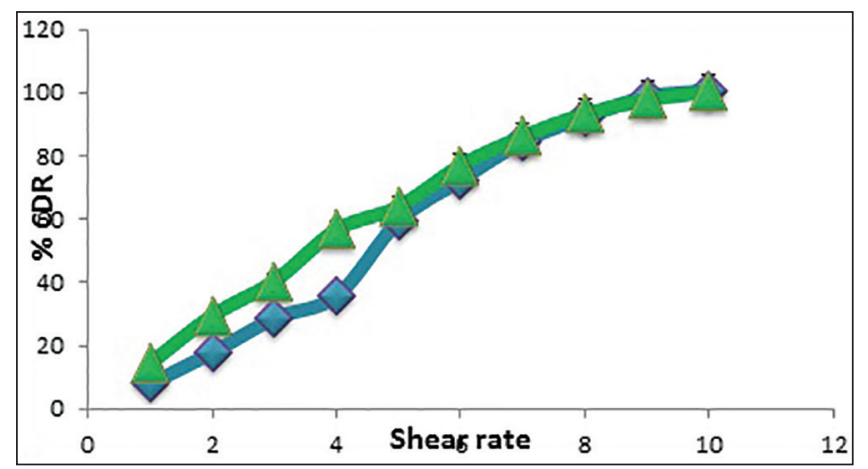

Figure 7: Release data after stability study of optimized batch (f-4) 
mechanical properties of clindamycin loaded in situ formulations. Bioadhesive polymer HPMC presumed to provide better vaginal bioadhesion. A low viscosity product may leak out of the vaginal cavity and too high viscous may interact with sexual intercourse. From present investigation it can be concluded that clindamycin gel formulating system can be successfully formulated by using combination of HPMC and gellan gum. The formulation is isotonic, easy to administer along with good bioadhesion and retention property. This formulation has potential for better patient compliance as vaginal formulation. The efficacy of the formulation can further be studied by in vivo and clinical experiments. As a result, the evaluation of the entire candidate formulations indicated that vaginal for mulation of clindamycin will be a new alternative for the treatment of vaginal candidiasis with suitable textural and rheological properties. Our results showed that the developed formulations were found worthy for further studies.

\section{REFERENCES}

1. William R, Vaginal Microbicide Formulation Workshop, Contraceptive Research and Development Program Arlington, Virginia; 1998.

2. Merabet J, Thompson D, Saul Levinson R. Advancing vaginal drug delivery. Expert Opin Drug Deliv 2005;2:769-77.

3. Chopra S, Motwani SK, lqbal Z, Talegaonkar S, Ahmad FJ, Khar RK. Optimisation of polyherbal gels for vaginal drug delivery by Box-Behnken statistical design. Eur J Pharm Biopharm 2007;67:120-31.

4. Patel PV, Vachanni MV. Biodegradable polymers-excipients for injectable drugs. Invent Rapid Nov Excip 2013;4:1-4.

5. Jain SK, Jain NK, Gupta Y, Jain A, Jain D, Chaurasia M. Mucoadhesive chitosan microspheres for non-invasive and improved nasal delivery of insulin. International Journal of Pharmaceutics 2007;64:498-504.
6. Gupta H, Sharma A. Ion activated bioadhesive in situ gel of clindamycin for vaginal application. Int J Drug Deliv 2009;10:32-40.

7. Patel PV, Koyani V. Smart polymers: Innovative drug delivery system. World J Pharm Pharm Sci 2014;3:508-27.

8. Velpandian T, Bankoti R, Humayun S, Ravi AK, Kumari SS, Biswas NR. Comparative evaluation of possible ocular photochemical toxicity of fluoroquinolones meant for ocular use in experimental models. Indian J Exp Biol 2006;44:387-91.

9. Spielmann H. Ocular irritation. In: Castle JV, Gomez MJ, editors. In Vitro Methods in Pharmaceutical Research. 2ed. San Dego, CA: Academic Press; 1997. p. 265-87.

10. Hatefi A, Amsden B. Biodegradable injectable in situ forming drug delivery systems. J Control Release 2002;80:9-28.

11. Vernon BL, Fusaro F, Borden B, Roy KH. Partition-controlled progesterone release from waterborne, in situ-gelling materials. Int J Pharm 2004;274:191-200.

12. 2 Hussain $A$, Ahsan $F$. The vagina as a route for systemic drug delivery. J Control Release 2005;103:301-13.

13. Kim EY, Gao ZG, Park JS, Li H, Han K. rhEGF/HP-beta-CD complex in poloxamer gel for ophthalmic delivery. Int $\mathrm{J}$ Pharm 2002;233:159-67.

14. Rashad AL, Toffler WL, Wolf N, Thornburg K, Kirk EP, Ellis G, et al. Vaginal PO2 in healthy women and in women infected with Trichomonas vaginalis: Potential implications for metronidazole therapy. Am J Obstet Gynecol 1992;166:620-4.

15. Owen DH, Katz DF. A vaginal fluid simulant. Contraception 1999;59:91-5.

16. Mortazavi SA, Carpenter BG, Smart JD. An investigation of the rheological behaviour of the mucoadhesive/mucosal interface. Int J Pharm 1992;83:221-5.

How to cite this article: Patel P, Patel P. Formulation and evaluation of clindamycin HCL in situ gel for vaginal application. Int J Pharma Investig 2015;5:50-6.

Source of Support: Nil. Conflict of Interest: None declared. 